\title{
indexGIXS - software for visualizing and interactive indexing of grazing-incidence scattering data
}

\author{
Detlef-M. Smilgies ${ }^{\mathrm{a} *}$ and Ruipeng $\mathrm{Li}^{\mathrm{b}}$
}

Cornell High Energy Synchrotron Source (CHESS), Cornell University, Ithaca NY 14853

${ }^{a}$ current address: Center for Advanced Microelectronics Manufacturing (CAMM) and Materials Science and Engineering Program, Binghamton University, Binghamton, NY 13902

${ }^{\mathrm{b}}$ current address: National Synchrotron Light Source II, Brookhaven National Laboratory, Upton, NY 11973

*corresponding author email: $\underline{\text { dms79@ } @ \text { cornell.edu }}$

\begin{abstract}
Grazing incidence small- and wide-angle scattering (GISAXS, GIWAXS) are widely applied for the study of organic thin films, be it for the characterization of nanostructured morphologies in block copolymers, nanocomposites, or nanoparticle assemblies, or the packing and orientation of small aromatic molecules and conjugated polymers. Organic thin films typically are uniaxial powders, with specific crystallographic planes oriented parallel to the substrate surface. The associated fiber texture scattering patterns are complicated by refraction corrections and multiple scattering. We present an interactive graphics tool to index such patterns.
\end{abstract}




\section{Introduction}

With the popular use of grazing-incidence x-ray scattering techniques for applications in nanoscience and organic electronics, there is an increasing demand for graphical software tools, in order to index scattering patterns interactively. Moreover, such a program should run on a flexible and low-cost platform that can be installed conveniently under the most common operating systems. The free and open source Scilab software (www.scilab.org) provides such a platform: it features a high-level programming language for easy manipulation of matrices, as well as powerful graphics and an easy-to-use graphical user interface. In the following we describe the interactive indexGIXS program for data visualization and indexing which is based on our earlier work on the indexing problem (Smilgies \& Blasini, 2007).

\section{Program description}

\subsection{Area detector data display}

indexGIXS reserves a large part of its application window for the detailed display of the measured scattering patterns. All types of area detectors in use at CHESS are supported (MedOptics, FLIcam, Dectris Pilatus 100k, 200k, and 300k, GE Typhoon 7000 image

plate reader). It is also possible to read in data from G2 reciprocal space maps employing a linear diode array in conjunction with Soller slits mounted on a two-axis detector arm (Smilgies et al., 2005; Nowak et al., 2006). The specifics of the chosen detector, such as pixel size and number, TIFF data format, and typical intensity range, can be displayed in the det_info menu by clicking the detector button. It is possible to extend the range of supported detectors. Other detectors using the TIFF format can be added directly choosing custom. Due to hardware and software limits, a maximum image size of $3 \mathrm{MB}$ should not be exceeded; for larger detectors binning can be used. 
When a detector is chosen, data can be read in using the load button. indexGIXS reserves a large part of its application window for the detailed display of the measured scattering patterns. The display of data can be further refined by specifying ranges in intensity and image size. Presets corresponding to the detector type are preloaded when reading the image. There is a choice of linear and logarithmic display (logscale) as well as providing a proper aspect ratio for rectangular detectors such as the Pilatus (isoview); alternatively the plot can be maximized on the available area, if isoview is unchecked. Image units are given in pixels or scattering vector $q$ for area detectors, or in degrees for reciprocal space maps (use units). The $q$-transformation is limited to the small-angle approximation and is meant for publication purposes while indexing should be done in pixel space.

\begin{tabular}{|c|c|c|c|c|c|c|c|c|c|}
\hline \multirow{3}{*}{ load } & rescale & mil & 15 & $\max$ & 15000 & & log scale & detector typ & \\
\hline & \multirow{2}{*}{ resiz } & \multirow{2}{*}{$\begin{array}{l}x \min \\
z \min \end{array}$} & -2.5 & \multirow{2}{*}{$\begin{array}{l}x \max \\
z \max \end{array}$} & 0.2 & & isoview & MedOptics & \\
\hline & & & 0 & & 2.5 & 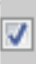 & use units & FLI Cam & \\
\hline
\end{tabular}

C:IUserslspecuser.CHESSIDocuments\My Dropboxld-linelGIXSpackdevlimages \PtCu-021210-c

Fig. 1. Data file input and manipulation menu. The "detector type" pull-down menu provides a list of supported detectors. The "detector type" push button displays the default settings for the supported detectors or permits the user to define a custom detector. When a detector type is chosen in the list box below, images can be loaded. The image file name is displayed below the menu. The "rescale" and "resize" push buttons in conjunction with the adjacent check boxes permit the user to fine-tune the display parameters (see text).

\subsection{Detector space versus reciprocal space}

In order to keep processing time at a minimum, it is most efficient to calculate positions of diffraction spots in detector space (pixels for area detectors, angles for reciprocal space maps), rather than perform the non-linear transformation of all pixels in the raw data to 
reciprocal space. This issue is most pronounced for GIWAXS; in GISAXS q-space is essentially a linear function of the scattering angles, and reciprocal space maps can be generated in good approximation by a linear transformation.

Even in the realm of small-angle scattering, there is still an advantage of remaining in detector space, which is due to $\mathrm{x}$-ray refraction and dynamic effects that will discussed in section 2.3. After all, of interest is the scattering vector inside the film, i.e. including refraction and reflection effects. Refraction corrections are essential for the interpretation of GISAXS data (Busch et al., 2006). These conditions are automatically detected in indexGIXS (see section 2.4 for details). Finally there are the diffuse scattering intensities close to the beam stop, where Bragg's law cannot be fulfilled and the Ewald sphere only cuts through the diffuse tails of the proper Bragg reflections (Smilgies 2019). With all these complications, we found it useful to simulate detector patterns rather than convert detector images to $q$-space, and then facing the problem of detangling the various scattering processes.

\subsection{Scattering pattern simulation}

The parameter set for specifying a GIXS pattern is quite elaborate and the menu, divided into logical submenus, is displayed to the right of the data. When the real-space lattice parameters are provided, the reciprocal lattice parameters can be determined (Authier, 2001). Using the Busing-Levy transform (Busing \& Levy 1967), the three dimensional lattice vectors are calculated (Smilgies \& Blasini, 2007).

The index rules pertaining to the space group can put in directly using the Bravais lattice types (primitive (P), centered (C), body centered (I), or face-centered (F)); additional screw axes and glide planes may be specified in the space group menu accessed by clicking the "index rule" push button, which is useful for small molecule lattices covering the triclinic, monoclinic and orthorhombic systems. The index rules menu also permits the user to set a limit for the maximum $h k l$ values used in the calculation, as well as to choose whether reflections are calculated for the left $(\mathrm{L})$, right $(\mathrm{R})$ or both $(\mathrm{B})$ sides 
of the incident plane. Common high-symmetry lattices such as SC, FCC, BCC, HCP, and diamond (DIA), as well as common block copolymer structures gyroid (GYR), hexagonal cylinders (cyl) and lamellae (lam) are generated automatically, if the $a$ lattice parameter is specified. The predefined lattice types are most useful when it is clear that simple lattices were formed, in particular for block copolymers (Paik et al., 2010; Chavis et al., 2015), mesoporous structures and nanocomposites (Crossland et al., 2009), as well as nanocrystal superlattices (Bian et al., 2011; Zhang et al, 2012).

A typical feature of thin films is that there is a preferential lattice plane formed parallel to the substrate surface. The Miller indices $H K L$ of this crystallographic plane have to be specified explicitly. For the pre-defined high-symmetry lattices the close-packed planes are assumed to form parallel to the substrate. Note that calculations are only performed, when hitting the " $G O$ ” button; hence even when starting with a high-symmetry lattice, lattice parameters and parallel planes can still be further modified before the calculation. With given lattice parameters and the parallel plane indices, the lattice vectors corresponding to this specific texture are generated (Smilgies \& Blasini, 2007).

A special role is played by the $s$-factor where " $s$ " stands for both swelling and shrinking. Thin films processed from solution or annealed in solvent vapor display a common feature that lattice spacings perpendicular to the substrate tend to expand during exposure to solvent vapor or shrink during drying, often past the equilibrium high-symmetry lattice. This non-equilibrium effect is pronounced in block copolymers with up to $40 \%$ swelling or shrinkage [see for instance (Crossland et al., 2009; Paik et al. (2010)], but occurs also to a lesser degree in nanoparticle assemblies (2-3\%). While such lattices display a lower symmetry in a crystallographic sense, it is still useful, to compare them to the high-symmetry equilibrium phase. In particular, it is easier to find an indexation starting from a high-symmetry phase with a close-packed plane parallel to the substrate. The $s$-factor is implemented as multiplying the 3D lattice vectors with the matrix $S$ : 


$$
S=\left(\begin{array}{ccc}
1 & 0 & 0 \\
0 & 1 & 0 \\
0 & 0 & 1 / f
\end{array}\right)
$$

where $f$ denotes the relative change of the perpendicular d-spacing as compared to the ideal high-symmetry crystal lattice.

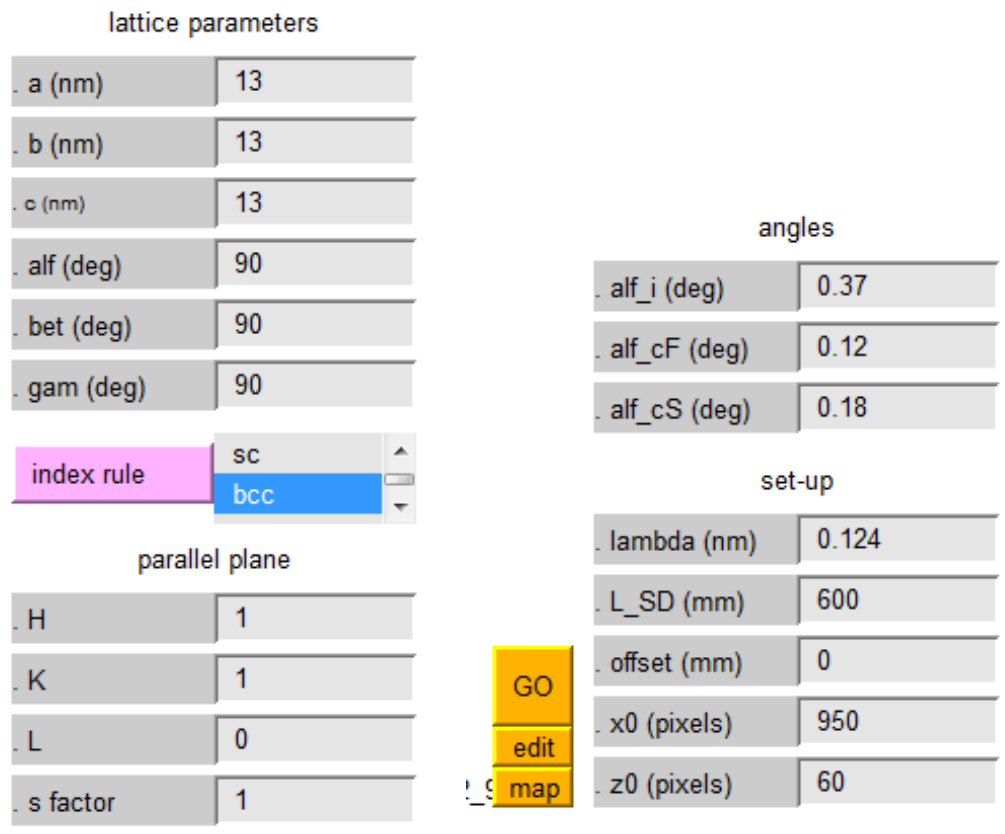

Figure 2. Input of lattice and set-up parameters. For convenience, all relevant parameters are displayed. On the left, the top part of the side bar with lattice parameters ( $a, b, c$, alf, bet, gam), index rule pull-down menu, and index of the texture plane and the s factor. On the right the lower part of the sidebar with angle menu and set-up menu. The “GO” pushbutton initializes the calculation. Numerical values can be inspected with "edit". "map" displays a map with reflections and indices to preview and correct spot assignments.

Other input parameters include the incident angle (alf_i) and the critical angles of substrate $\left(a l f \_s S\right)$ and thin film $\left(a l f \_c F\right)$. Finally the experimental parameters such as the X-ray wavelength (lambda) and the sample-to-detector distance $\left(L \_S D\right)$ can be specified. Furthermore the pixel positions of the direct beam on the detector $(x 0, z 0)$ need to be 
provided. The offset parameter is used, in case the detector is shifted horizontally from the position where the direct beam position was measured.

\subsection{Refraction correction}

The actual scattering process of interest takes place inside the thin film (Busch et al., 2006; Breiby et al., 2008). Hence for grazing incidence scattering, the refraction of incident and exit wave have to be accounted for, as incident angle and scattering angles are close to the critical angles of the materials. The incident wave vector inside the film $k_{i}^{\prime}$ can be decomposed into the parallel component which is continuous across the interface and a perpendicular component $k_{i z}^{\prime}$ which is related to the vacuum wave vector $k_{0}$ by

$$
k_{i z}^{\prime}=k_{0} \sqrt{\sin ^{2}(\alpha)-\sin ^{2}\left(\alpha_{c F}\right)}
$$

where $\alpha$ and $\alpha_{c F}$ denote the incident angle and the critical angle of the film, respectively.

If there is a scattering process with scattering vector $\boldsymbol{q}$ and vertical component $q_{z}$ inside the film, the $\mathrm{z}$-component of the scattered wave vector inside the film is given by

$$
k_{f z}^{\prime}=k_{i z}^{\prime}+q_{z}^{\prime}
$$

In case the incident angle lies between the critical angles of substrate and film, or more precisely, if $\alpha_{c F}<\alpha_{i}<\alpha_{c S}$, then the incident wave gets reflected at the substrate and the reflected wave has a perpendicular component of the wave vector

$$
k_{r z}^{\prime}=-k_{i z}^{\prime}
$$

The reflected wave is then scattered in the film resulting in another scattering spot at 


$$
k_{r f z}^{\prime}=k_{r z}^{\prime}+q_{z}^{\prime}=q_{z}^{\prime}-k_{i z}^{\prime}
$$

i.e. all reflections appear to be doubled up (Lee et al., 2005, Busch et al., 2006; Breiby et al., 2008). In an experiment it is very important to distinguish between this "double vision" effect due to the scattering process and a sample effect, for instance in case the sample is featuring two preferential orientations with two closely spaced $q$-vectors. A classic example for the latter are the thin film and bulk phases of pentacene (Dimitrakopoulos \& Malenfant, 2002; Nabok et al., 2007; Amassian et al. 2010). A convenient way of separating the two effects is to analyze the incident angle dependence. In the case of "double vision" the splitting vanishes below the film critical angle and increases until the substrate critical angle is reached and then the reflection with the higher $q z$ value vanishes beyond the substrate critical angle. All reflections, including higher orders, show the same splitting. In contrast, for the case of two similar structures the splitting is independent of the incident angle (except for a minor refraction correction). Moreover for higher order reflections the splitting is increased proportional to the order of the reflection pairs.

For the scattered wave (or waves) the refraction correction has to be applied again, when exiting the film:

$$
k_{i z}^{\prime}=k_{0} \sqrt{\sin ^{2}(\beta)+\sin ^{2}\left(\alpha_{c F}\right)}
$$

and similarly for the reflected wave.

If the exit angle $\beta$ coincides with the critical angles of the film $\alpha_{c F}$, the scattering intensity will peak which corresponds to the well-known Yoneda-Vinyard effect (Yoneda 1963; Vinyard 1982). For scattered waves with exit angles between the critical angles of film and substrate commonly a band of enhanced intensity is observed in the detector images which we have termed the "Yoneda band". For high-quality films with constant 
thickness and smooth surfaces, wave guide resonances can appear inside the Yoneda band yielding further intensity enhancement (Wang et al 2010). Beyond the substrate critical angle the scattering will behave increasingly kinematic, however the refraction correction remains essential to obtain correct d-spacings (Busch et al., 2006) and sfactors. indexGIXS will automatically detect the "double vision" condition and calculate single or double diffraction spots accordingly.

\subsection{Pseudo z-axis scattering geometry}

The pseudo-z-axis scattering geometry is a very efficient and easy to implement scattering geometry in the practical use at a beamline, for both GISAXS or GIWAXS area detector set-ups as well as for grazing-incidence reciprocal space mapping (Smilgies et al., 2005). In this geometry, rather than tilting the whole goniometer or bending the beam down, as used in the proper z-axis scattering geometry (Bloch 1985), only the sample is tilted, while the detector remains aligned perpendicular to the incident beam in the case of area detectors, or in the case of reciprocal space mapping the in-plane and outof-plane detector arm motions are centered on the direct beam. The pseudo z-axis geometry is compatible with most transmission SAXS and WAXS set-ups, so that such beamlines can be adapted to support grazing-incidence scattering experiments as well. 


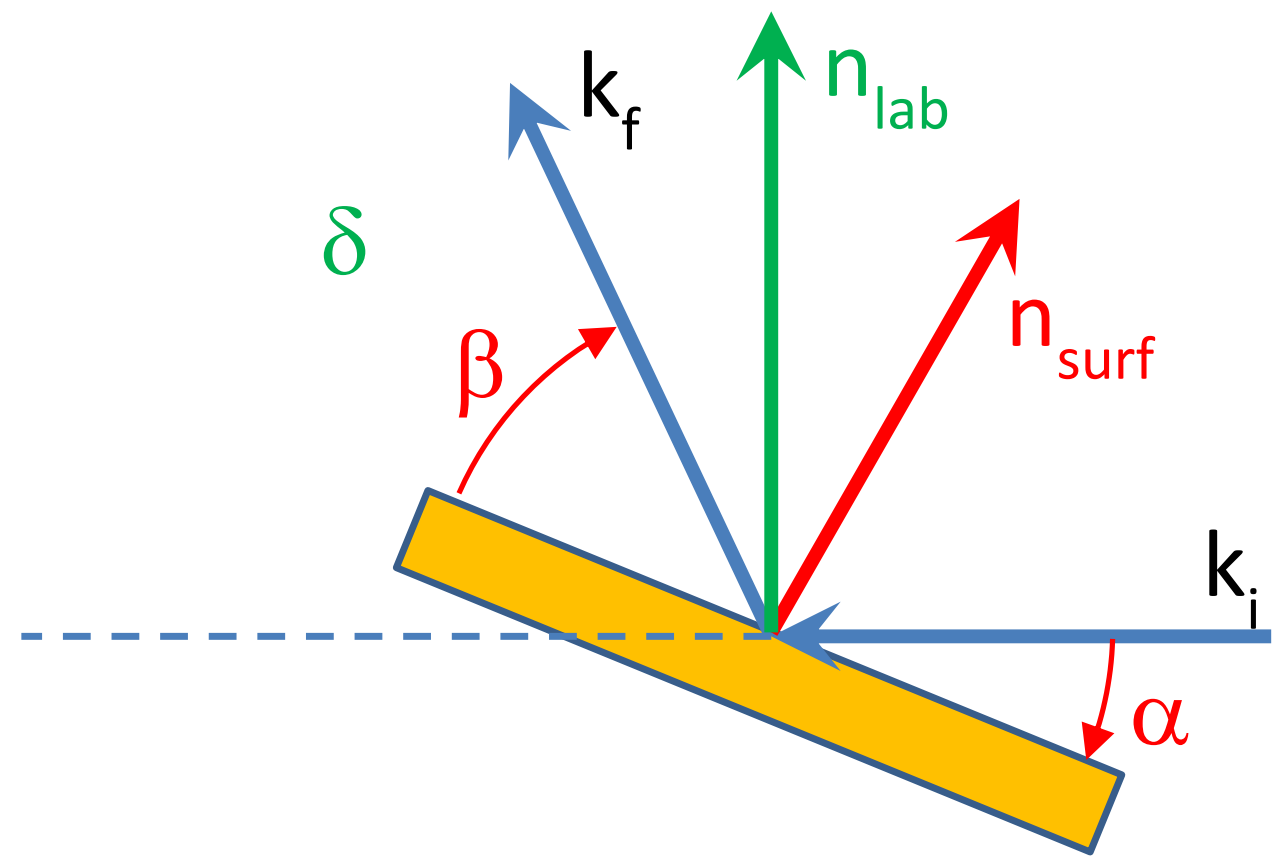

Fig. 3 Pseudo z-axis scattering geometry in the incident plane showing wave vectors, as well as the surface normal and scattering angles in the surface (red) and the lab frames (green). The sample is tilted by the incident angle $\alpha$.

The question is now, how are the $x$ and $z$ coordinates for an area detector or, alternatively, the equatorial $(v)$ and meridional $(\delta)$ detector arm angles of a $2+2$ diffractometer (Evans-Lutterodt \& Tang, (1995); Renaud et al. (1995)) or psi-axis diffractometer (You 1999) related to the natural surface scattering angles $\alpha, \psi, \beta$ (Smilgies \& Blasini, 2007).

The direction of the incident wave vector $\widehat{\boldsymbol{k}}_{\boldsymbol{i}}$ be given as the $y$ direction, and the vertical direction $\widehat{\boldsymbol{n}}$ of the laboratory system as the $z$-axis.

$$
\hat{\mathbf{k}}_{i}=\left(\begin{array}{c}
0 \\
1 \\
0
\end{array}\right) \quad \hat{\mathbf{n}}=\left(\begin{array}{l}
0 \\
0 \\
1
\end{array}\right)
$$


For the exact calculation we introduce the rotational matrices for rotations about the $x$ axis and the $z$-axis in the laboratory system, where the $x$-axis is horizontal and perpendicular to the $\mathrm{x}$-ray beam:

$$
\mathbf{R}_{x}(\varphi)=\left(\begin{array}{ccc}
1 & 0 & 0 \\
0 & \cos (\varphi) & -\sin (\varphi) \\
0 & \sin (\varphi) & \cos (\varphi)
\end{array}\right) \quad \mathbf{R}_{z}(\phi)=\left(\begin{array}{ccc}
\cos (\varphi) & -\sin (\varphi) & 0 \\
\sin (\varphi) & \cos (\varphi) & 0 \\
0 & 0 & 1
\end{array}\right)
$$

The direction of the scattered wave vector can now be related to the natural surface scattering angles using:

$$
\hat{\mathbf{k}}_{f}=\mathbf{R}_{x}(\alpha) \mathbf{R}_{z}(\psi) \mathbf{R}_{x}(\beta) \hat{\mathbf{k}}_{i}
$$

The corresponding $\delta$ angle of the psi-circle diffractometer is obtained from the scalar product of $\hat{\mathbf{k}}_{f}$ and $\hat{\mathbf{n}}$, which is simply the $z$-component of $\hat{\mathbf{k}}_{f}$ :

$$
\sin (\delta)=\cos (\pi-\beta)=\hat{\mathbf{n}} \cdot \hat{\mathbf{k}}_{f}=\hat{k}_{f z}
$$

Evaluation of the matrix multiplications yields

$$
\sin (\delta)=\sin (\alpha) \cos (\psi) \cos (\beta)+\cos (\alpha) \sin (\beta)
$$

For $\psi=0$ this yields the exact result $\delta=\beta+\alpha$. For small $\alpha$ and $\beta$ the sine function can be expanded to first order and we obtain the small-angle approximation (Smilgies \& Blasini, 2007):

$$
\delta=\beta+\alpha \cos (\psi)
$$

This approximation holds even for larger $\beta$ and $\psi$ within a couple of $0.1^{\circ}$. 
In a similar fashion we can find the $v$ angle:

$$
\tan (v)=\hat{k}_{f x} / \hat{k}_{f y}
$$

which evaluates to

$$
\tan (v)=\frac{\sin (\psi) \cos (\beta)}{\cos (\alpha) \cos (\psi) \cos (\beta)-\sin (\alpha) \sin (\beta)}
$$

For small $\alpha$ and $\beta$ we retrieve again the small-angle approximation (Smilgies \& Blasini, 2007):

$$
v=\psi
$$

However, the residual error for both $\psi$ and $\beta$ larger than $10^{\circ}$ becomes on the order of $1^{\circ}$. Hence for wide-angle scattering it is advisable to use the exact formula. The indexGIXS program uses the exact formulae for both the GISAXS and GIWAXS regimes.

Knowing the diffractometer angles $v$ and $\delta$, it is now straightforward to obtain the locations $x, z$ on a planar area detector relative to the point where the scattered $\mathrm{x}$-ray beams would hit. With the plane of the detector perpendicular to the incident beam and the sample-detector distance is $L$, we obtain:

$$
\begin{aligned}
& x=L \tan (v) \\
& z=L \tan (\delta) / \cos (v)
\end{aligned}
$$

These detector positions can then be converted to pixel values, and knowing the pixel location of the direct beam the calculated scattering pattern can be superimposed to the raw data. 


\section{Use of the Program}

\subsection{Calibration of the set-up}

The first information on a given set-up to obtain is the wavelength of the x-ray beam and in the case a small- or wide-angle scattering the sample-detector distance $L$; the latter is often determined with a standard, for example silver behenate for small angle scattering and silicon or ceria powder for wide-angle scattering. Finally the position of the direct beam on the detector needs to be determined, taken with an appropriate attenuator.

\subsection{Refraction parameters}

Typically the incident angle of the x-ray beam is determined during sample line-up. The critical angles of the film and the substrate can be measured, if line up is combined with a reflectivity measurement covering the angle range from 0 to at least $2 \alpha_{c s}$. The substrate critical angle can often also be calculated from the known optical constants of the substrate. In in-situ experiments such a solvent vapor processing (Posselt et al., 2017), it can happen that the sample moves. Below we describe a simple procedure in which the angular information can be recovered in such a case. This correction is advisable, as the precision of $s$-factor (see section 2.1) is affected by imprecise refraction parameters. 


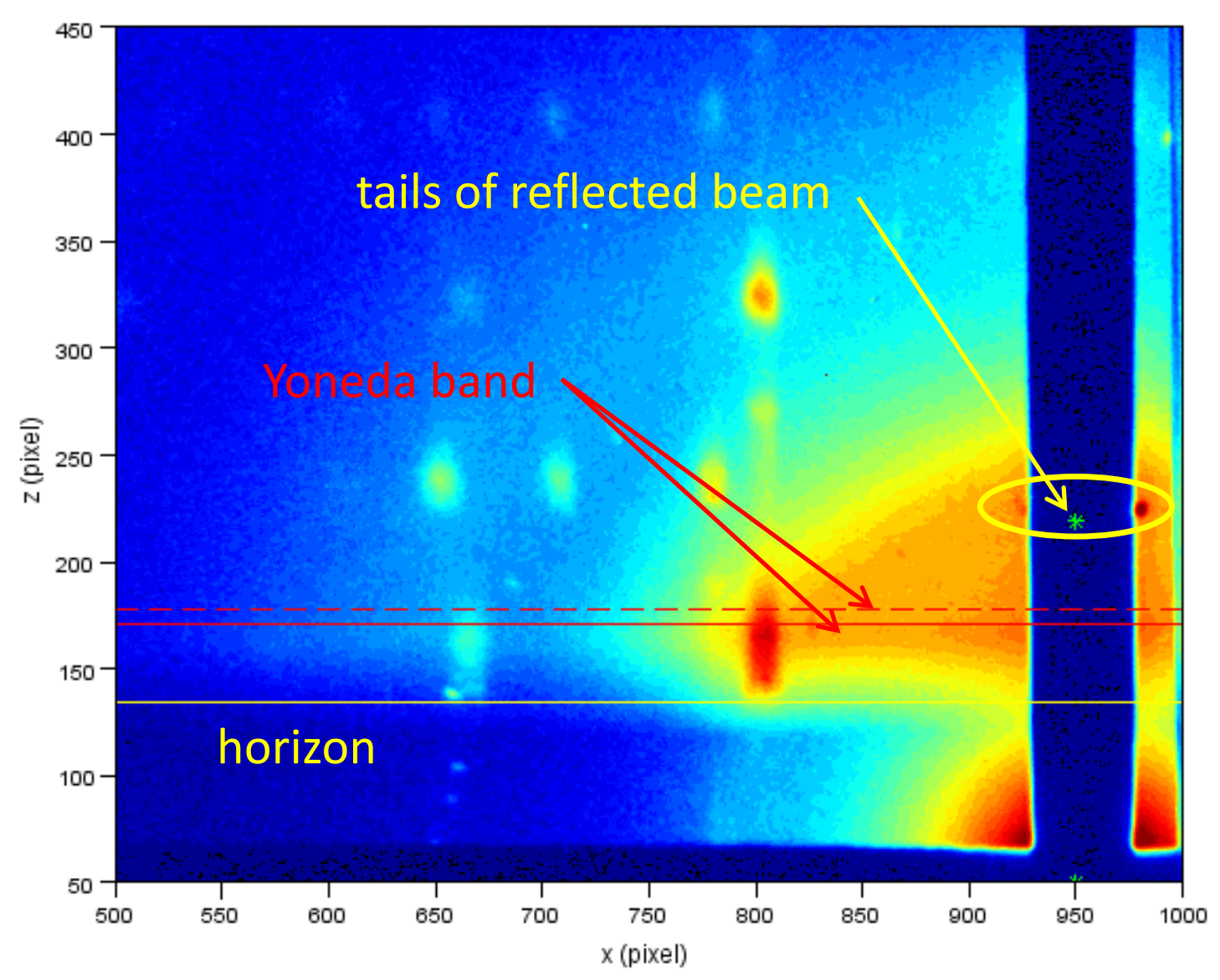

Fig. 4. Revision of refraction parameters: direct and reflected beam are indicated by stars (green). Often tails of the reflected beam can be discerned close to the beamstop. A small increase in background scattering intensity indicates the position of the horizon (solid yellow line). Both reflected beam tails and horizon are solely dependent on the incident angle. The Yoneda band, the band of enhanced scattering intensity between the critical angle of the film and the substrate helps to revise the optical parameters. The film Yoneda line is found at the first maximum of the scattering intensity coming from low $z$ (solid, red line). The substrate Yoneda line appears often only as a shoulder (dashed red line). Usually calculation from the substrate optical constants results in a good fit. 
The refinement of the refraction parameters should proceed along the following steps:

- Refine the incident angle so that the diffuse tail of the reflected beam close to the beamstop comes out correctly.

- If the position of the reflected beam is not clear, adjust the incident angle to get the sample horizon (at $\beta=0$, indicated by the yellow line) and the lower edge of the Yoneda band right.

- The critical angle of the substrate can be obtained from the CXRO on-line calculator (CXRO, ) knowing the beam energy.

- Then refine the film critical angle by fitting the intensity maximum at the lower edge of the Yoneda band.

At this point the refraction parameters are established $\left(\alpha_{i}, \alpha_{c F}, \alpha_{c S}\right)$.

\subsection{In-plane lattice}

Next the lattice constants in the surface plane are to be established. These are not affected be refraction or dynamic effects, however, are the result of a $2 \mathrm{D}$ powder. So for instance

a hexagonal in-plane lattice would yield the tell-tale series $1, \sqrt{3}, \sqrt{4}, \sqrt{7}, \ldots$ if all parallel components of the reflections are taken into account. Next the lattice constants have to be established. If there is already an expectation with regards to the lattice type (FCC or $\mathrm{HCP}$ ) one can start from there with a bit of trial and error to fit the in-plane lattice. Otherwise one can use a simple hexagonal lattice as starting point to establish the inplane lattice constant. For lattices of low symmetry, such as GIWAXS of molecular lattices, a known bulk structure of the molecule or a related molecule can provide a good starting point.

\subsection{Out-of-plane lattice and shrinkage factor}

Again the example of the in-plane hexagonal lattice is quite revealing, on how to proceed: an in-plane hexagonal lattice can be due to $\mathrm{FCC}(111)$ and $\operatorname{HCP}(001)$, but also simple hexagonal; even $\mathrm{BCC}(110)$ is only a slightly distorted hexagonal lattice. In order to discern between these lattices, the index rules come into play - the pattern of allowed 
and forbidden reflections. For instance FCC(111) features ABC stacking, hence there are 2 reflections between the diffuse projections of the main series reflections close to the beam stop. $\mathrm{HCP}(001)$ and $\mathrm{BCC}(110)$ feature $\mathrm{AB}$ stacking, and thus there is only one reflection in between main series reflections. For simple hexagonal or cubic lattices with (001) orientation all reflections are in lock-step in the z-direction corresponding to simple AA stacking. If main series reflections are not visible - often a sign of a highly ordered film - then we will have to go by the pattern of allowed and forbidden reflections that the program calculates based on the Bravais type of the lattice.

Low-symmetry lattices of molecules tend to be much harder to index. Starting from a known bulk structure can provide a good starting point. The parallel plane orientation can be either established, if there is a $\theta-2 \theta$ scan available, or if molecular visualization software such as Mercury by the Cambridge Crystallographic Database can help to identify close-packed planes. Molecular lattices feature a large range of scattering intensities, and it is not always clear whether a missing spot is due to a weak reflection or space group symmetry. indexGIXS provides additional space group elements to use with the Bravais lattices, such as glide planes and screw axes, which help to determine whether systematic absences do occur.

The final step is to refine the shrinkage/swelling parameter so that the perpendicular spot positions are matched. Note: the accuracy of this step is determined by having determined the refraction parameters correctly. As refraction effects are more pronounced close to the Yoneda band, one should start fitting the reflection at higher exit angle. If discrepancies remain, this is often an indication that the beam or the refraction parameters need more fine tuning. If all falls into place, the full lattice including lattice type and parameters, parallel plane, and shrinking parameter have been established, along with the critical angles of the film. 


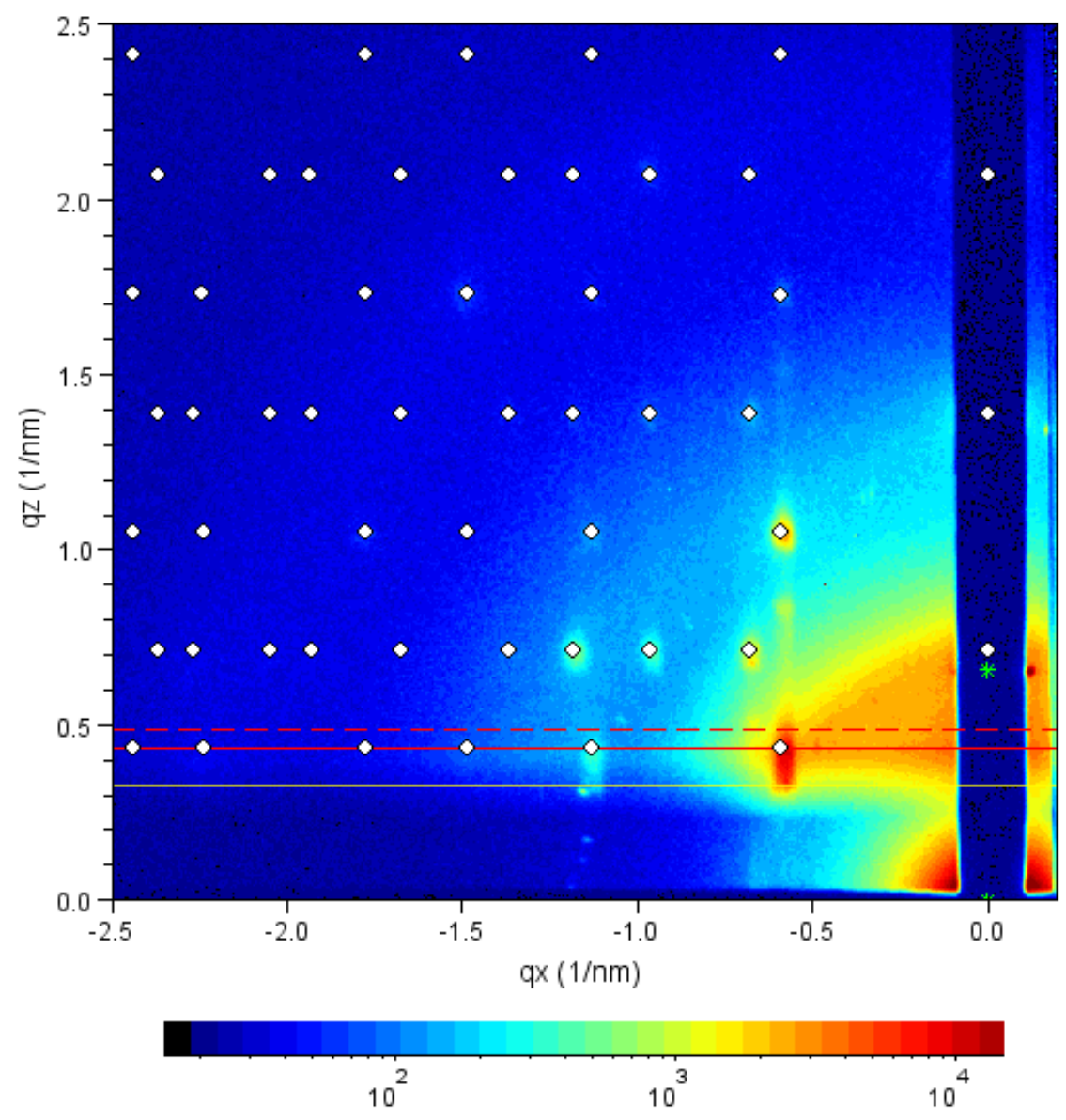

Figure 5. Indexing a BCC superlattice of octahedral Pt ${ }_{2} \mathrm{Cu}_{3}$ nanoparticles. Publicationstyle indexGIXS output was generated for the SAXS regime. (GISAXS data from D1 beamline)

For a more elaborate application we show the indexing of the wide-angle scattering pattern of a thin film of pyrene deposited on a glass slide. In this case the $\mathrm{G} 2$ psi-axis diffractometer was used for reciprocal space mapping using a linear diode array as detector. The $v$ angle was scanned while the $\delta$ angle was determined by the calibration of the linear detector. Pyrene crystallizes in the monoclinic space group P2 1 /a (Kai et al., 
1978). Hence in the index rule menu the unit cell was chosen as primitive $(\mathrm{P})$ and in addition an index rule for the a glide plane (denoted "b/a") was chosen in the index rule menu. The label of the pushbutton is changed to a red color, in order to indicate that a space group element was invoked. After lattice parameters, space group, and instrument parameters were set, it could be established that pyrene crystallized in polymorph I with the (100) plane parallel to the substrate. This being a known bulk polymorph, the known crystal structure can be used to establish, how the molecules are oriented with regard to the substrate surface.

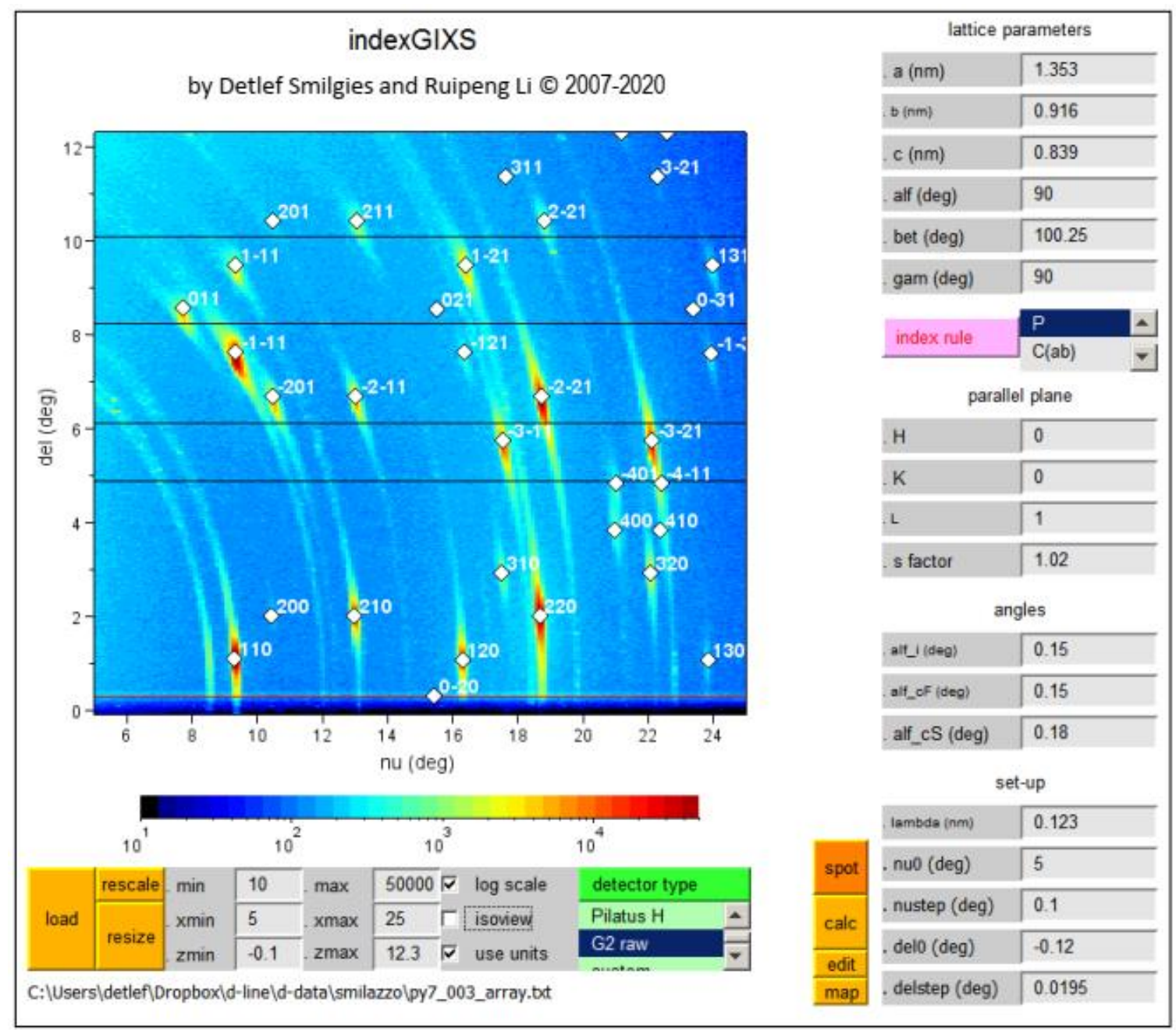

Figure 6. Indexing the monoclinic lattice of pyrene from a reciprocal lattice map obtained with a linear diode array mounted on a psi-circle diffractometer. The dark lines are due to dead elements in the diode array. The successful indexation with a known bulk 
polymorph shows that pyrene assumes the room-temperature bulk structure with the (100) plane parallel to the substrate surface.

The indexing of thin film diffraction and scattering patterns shown here relies on known bulk structures or high symmetry-lattices and was essentially done by trial and error. Nonetheless indexGIXS has been successfully applied by a number of other users apart from ourselves to determine lattice type, polymorph, and lattice orientation in crystalline and nanostructured thin films. Recently more sophisticated computer-based approaches have emerged that automate the thin film lattice structure determination, for instance (Mannsfeld et al., 2011; Hailey et al., 2014; Toney 2019; Kainz et al., 2021).

\subsection{Software requirements and distribution}

indexGIXS runs under the current Scilab 6.1.n distribution on Windows, Linux, and MacOS platforms. The Scilab software can be downloaded free of charge from www.scilab.org. indexGIXS has been prototyped and extensively tested as a MathCAD® script, was then ported to $M a t L a b{ }^{\circledR}$, and finally to the Scilab open source platform. The program is part of a suite of programs for the analysis of area detector data. indexGIXS is freely distributed by the corresponding author (1ms79@ cornell.edu) on request.

\section{Acknowledgement}

We are grateful to the CHESS users who have applied indexGIXS and have provided helpful feedback. Jun Zhang and James Fang, SUNY Binghamton, be thanked for the use of the scattering image of the $\mathrm{Pt}_{2} \mathrm{Cu}_{3}$ nanoparticle superlattice. We thank Stephanie Milazzo, Rhodes College, for expertly preparing the pyrene sample as part of her Research Experience for Undergraduates project. Part of this work was supported by the National Science Foundation under award DMR-1332208. 


\section{References}

Amassian, A., Pozdin, V. A., Li, R., Smilgies, D.-M. \& Malliaras, G. G. (2010). J. Mater. Chem. 20, 2623-2629.

Authier, A. (2001). IUCr Pamphlet Series, No. 4, The Reciprocal Lattice, http://www.iucr.org/iucr- top/comm/cteach/pamphlets/4/4.pdf.

Bian, K., Choi, J. J., Kaushik, A., Clancy, P., Smilgies, D.-M., \& Hanrath, T. (2011). ACS Nano 5, 2815-2823.

Bloch, J. M. (1985). J. Appl. Cryst. 18, 33-36.

Breiby, D. W., Bunk, O., Andreasen, J. W., Lemke, H. T. \& Nielsen, M. M. (2008). J. Appl. Cryst. 41, 262-271(2008).

Busch, P., Rauscher, M., Smilgies, D.-M., Posselt, D. \& Papadakis, C. (2006). J. Appl. Cryst. 39, 433-442.

Busing, W. R. \& Levy, H. A. (1967). Acta Cryst. 22, 457-464.

Chavis, M. A., Smilgies, D.-M., Wiesner, U. \& Ober, C. K. (2015). Adv. Funct. Mater. 25, 3057-3065.

Crossland, E., Kamperman, M., Nedelcu, M., Ducati, C., Wiesner, U., Toombes, G., Hillmyer, M., Ludwigs, S., Steiner, U., Smilgies, D.-M. \& Snaith, H. (2009). Nano Lett. 9, 2807-2812.

CXRO (retrieved 9/2021).https://henke.lbl.gov/optical_constants/

Dimitrakopoulos, C. D. \& Malenfant, P. R. L. (2002). Adv. Mater. 14, 99-117.

Evans-Lutterodt, K. \& Tang, M.-T. (1995). J. Appl. Cryst. 28, 318-326.

Feidenhans'1, R. (1989). Surf. Sci. Rep. 10, 105-188.

Hailey, A. K., Hiszpanski, A. M., Smilgies, D.-M. \& Loo Y.-L. (2014). J. Appl. Cryst. 47, 2090-2099.

Kai, Y., Hama, F., Yasuoka, N., Kasai, N. (1978). Acta Cryst. B 34, 1263-1270.

Kainz, M. P., Legenstein, L., Holzer, V., Hofer, S., Kaltenegger, M., Resel, R. \& Simbrunner, J. (2021). J. Appl. Cryst. 54, 1256-1267.

Lee, B., Park, I., Yoon, J., Park, S., Kim, J., Kim, K.-W., Chang, T. \& Ree, M. (2005). Macromolecules 38, 4311-4323.

Mannsfeld, S. C. B., Tang, M. L. \& Bao, Z. (2011). Adv. Mater. 23, 127-131. 
Nabok, D., Puschnig, P., Ambrosch-Draxl, C., Werzer, O., Resel, R. \& Smilgies, D.-M. (2007). Phys. Rev. B 76, 235322,

Nowak, D. E., Blasini, D. R., Vodnick, A. M., Blank, B., Tate, M. W., Deyhim, A., Smilgies, D.-M., Abruña, H., Gruner, S. M., \& Baker, S. P. (2006). Rev. Sci. Instrum. 77, 113301 (2006).

Paik, M. Y., Bosworth,J. K., Smilgies, D.-M., Schwartz, E. L., Andre, X. \& Ober, C. K. (2010). Macromolecules 43, 4253-4260.

Posselt, D., Zhang, J., Smilgies, D.-M., Berezkin, A.,Potemkin, I. I. \& Papadakis, C. M. (2017). Progr. Polymer Sci. 66 80-115.

Renaud, G., Villette, B. \& Guenard, P. (1995). Nucl. Instrum. Methods B, 95, 422-430.

Savikhin, V., Steinrück, H.-G., Liang, R.-Z., Collins, B. A., Oosterhout, S. D., Beaujuged, P. M. \& Toney, M. F. (2020). J. Appl. Cryst. 53, 1108-1129.

Smilgies, D.-M. (2002). Rev. Sci. Instrum. 73, 1706-1710 (2002).

Smilgies, D.-M., Blasini, D. R., Hotta, S. \& Yanagi, H. (2005). J. Synchrotron Rad. 12, $807-811$.

Smilgies, D.-M. \& Blasini, D. R. (2007). J. Appl. Cryst. 40, 716-718 (2007).

Smilgies, D.-M. (2019). J. Appl. Cryst., 52, 247-251.

Vineyard, G. H. (1982). Phys. Rev. B 26, 4146-4159.

Yoneda, Y. (1963). Phys. Rev. 131, 2010-2013.

You, H. (1999). J. Appl. Cryst. 32, 614-623.

Zhang, J., Luo, Z., Martens, B., Quan, Z., Kumbhar, A., Porter, N., Wang, Y., Smilgies, D.-M. \& Fang, J. (2012). J. Am. Chem. Soc. 134, 14043-14049. 